\title{
Using Neuropeptide $Y$ as a Marker to Track GABAergic Neurons in Rat Brains Prenatally Primed with Betamethasone
}

\author{
Akshara Koottala \\ New Rochelle High School, 265 Clove Rd, New Rochelle, NY, 10801, USA; aksharakoottala@gmail.com
}

ABSTRACT: Epilepsy is a common neurological disease with a strong genetic component. Epilepsy can be caused by the lack of GABAergic neurons which release $\gamma$-Aminobutyric acid (GABA), an inhibitory neurotransmitter responsible for regulating excitability in the central nervous system. It is not clear if the lack of these neurons in the cortex and striatum (two brain regions associated with several epilepsy syndromes) can be caused by prenatal exposure to a synthetic steroid, betamethasone. Therefore, we prenatally administered betamethasone to observe its effects on GABA production in rat brains. We used neuropeptide $Y$ (NPY) as a marker for GABA to measure a change in GABA expression in the cortex and striatum of male and female rats. Results revealed that prenatal betamethasone exposure led to a significant decrease of NPY in the cortex compared to the saline-primed control. Further analysis of the cortex indicated a statistically significant sex differences, demonstrating lower GABA counts in female offspring. The striatum, however, did not show a significant difference. Ultimately, this decrease in NPY positive GABAergic neurons may make the offspring more susceptible to having seizures and developing epilepsy. The results of this study establish a link between genetics and the development of epilepsy in offspring.

KEYWORDS: Biology; Neuroscience; Epilepsy; Transgenerational Epigenetic Inheritance; Cortex; Striatum; GABAergic Neurons; Neuropeptide Y.

\section{- Introduction}

Epilepsy is the fourth most common neurological disease today and it affects millions of people from all over the world and from every age demographic. ${ }^{1}$ It is a chronic condition characterized by recurrent, unprovoked seizures which are defined as frequent changes in the electrical activity of the brain that occur without stimulus. ${ }^{2}$ More than 25 epilepsy syndromes have been delineated that range in severity and seizure types, making it a spectrum of disorders. ${ }^{1}$ Despite it being such a predominant disease, the etiology of epilepsy still remains a predominant issue in the medical field. Numerous studies have all concluded that the causes of epilepsy each reflect an underlying brain dysfunction. ${ }^{3}$ In order to diagnose the cause of each specific form of epilepsy, recent studies have focused on neuronal activity in the brain controlled by the balance of neurotransmitters in the brain. These neurotransmitters, or chemical compounds responsible for communication between the neurons, ensure that cell excitability is regulated in order to prevent seizures. A decrease in expression of these neurotransmitters can cause an increase in spasms. ${ }^{4}$ According to previous studies, stress is one of the main causes for a decrease in expression of neurotransmitters because it directly alters the epigenome, where production of neurotransmitters is regulated by gene expression. Similar to the human condition, stress in rats also can lead directly to alterations that affect neurotransmitter production. Researchers exploring the alterations made to the epigenome have found recent evidence that suggests there may be genetic predisposition to developing the disease. ${ }^{5}$

Betamethasone as a Model Stressor:

As mentioned before, a deficiency in neurotransmitters caused by stress may significantly contribute to occurrence of seizures, which are the hallmarks of epilepsy. Considering 75\% of epilepsy begins during childhood, the developing brain is the most susceptible to epilepsy and the main reason could be the deficiency of neurotransmitters in the direct offspring of stressed mothers. ${ }^{3}$ One method of causing stress and anxiety in these mothers is by directly injecting drugs called corticosteroids. Corticosteroids are steroid hormones synthetically made to be given by injection to start anti-inflammatory processes. One of the drugs that have yet to be thoroughly researched with regards to epilepsy is betamethasone, a synthetic corticosteroid. Severe stress or significant exposure to betamethasone during pregnancy can predispose the offspring to development of infantile spasms (also known as West Syndrome or epileptic spasms). ${ }^{6}$ This is a form of epilepsy that predominantly occurs between 3 and 12 months. It is a catastrophic pediatric epilepsy with motor spasms, persistent seizures, mental retardation, and in some cases, autism. ${ }^{7}$ Velisek et al 2007 developed a rat model of cryptogenic infantile spasms by using prenatal exposure to betamethasone combined with a postnatal trigger of spasms with N-methyl-D-aspartic acid (NMDA). ${ }^{8}$ The general conclusion reached was that prenatal priming with betamethasone can lead to a deficiency in neurotransmitters, specifically GABAergic neurons. The lack of GABA neurons increased susceptibility of spasms and made the offspring more vulnerable to developing seizures and eventually epilepsy.

\section{The Relationship between Neuropeptide $Y$ and GABAergic Neurons:}

GABAergic neurons release $\mathrm{y}$-Aminobutyric acid (GABA) which is the main inhibitory neurotransmitter in the cerebral cortex. ${ }^{9}$ GABAergic inhibition allows for synchronization 
of activity in cortical networks, including gating of activity, controlling movements and dictating the manner in which activity flows. ${ }^{10} \mathrm{It}$ is a fundamental part of the brain because a blockade of GABA inhibition can lead to an increase in cell excitability and result in absence seizures with spike-and-wave discharges, eventually leading to epilepsy. ${ }^{11}$ These GABA neurons carry out their functions together with neuropeptides like neuropeptide Y(NPY), which are small protein-like molecules (peptides) that are used by neurons to communicate with one another NPY is the most abundant neuropeptide in the brain and serves as a marker for GABAergic neurons in the brain. It plays a major role in brain activity because it signals molecules that engage in many physiological functions. ${ }^{12} \mathrm{NPY}$ is crucial in the brain because a loss of these peptides signifies a decrease in GABA neurons which may disrupt the ability of the brain to filter incoming seizure activity. This eventually leads to neuronal injury and intractable seizures which can cause epilepsy. ${ }^{13}$

The two sections that have a dense NPY population and are associated with the development of epilepsy are the striatum and cortex. The striatum, a part of the basal ganglia, plays a significant role in facilitating voluntary movement, and consequently, is a major source of excitatory inputs and GABA neuron production. Similarly, the cerebral cortex is critical because the neuron activity in this region controls emotions, problem solving, critical thinking, the ability to plan, and the recognition of parts of speech. ${ }^{14}$ The main focus of many epileptic studies is the neuronal activity in the cortex region, specifically neocortex, because its low activity is indicative of increased susceptibility to seizures, especially in newborn infants..$^{15}$ These two regions are extremely important because a recent study using a mouse model of childhood epilepsy showed that epilepsy can be triggered by impaired communication between the cortex and striatum. ${ }^{11}$

Goal:

There is a gap in research regarding whether the prena tal administration of the stressor drug, betamethasone, will positively or negatively affect the production of GABAergic neurons in the cortex and striatum regions. The purpose of this study was to determine the effect of prenatal treatment with betamethasone on GABAergic neuron expression in offspring by counting the NPY positive cells (marker for GABA) in the striatum and cortex. We hypothesized that prenatal exposure to betamethasone will decrease the GABAergic neuron count in the cortex and striatum.

\section{Results and Discussion}

\section{GABAergic Count Based on Treatment:}

All statistics in the study were done using two-way ANOVA with factors of sex (levels males and females) and prenatal treatment (levels saline and betamethasone). Images obtained from immunohistochemistry staining clearly show a decrease in the population of NPY in rat brains with prenatal treatment of betamethasone. The mean count of NPY-expressing cells within the cortex sections from the stressed rats treated with betamethasone was 26.676 NPY cells/mil pix (cells in the cortex standardized per 1 million pixels) whereas the average

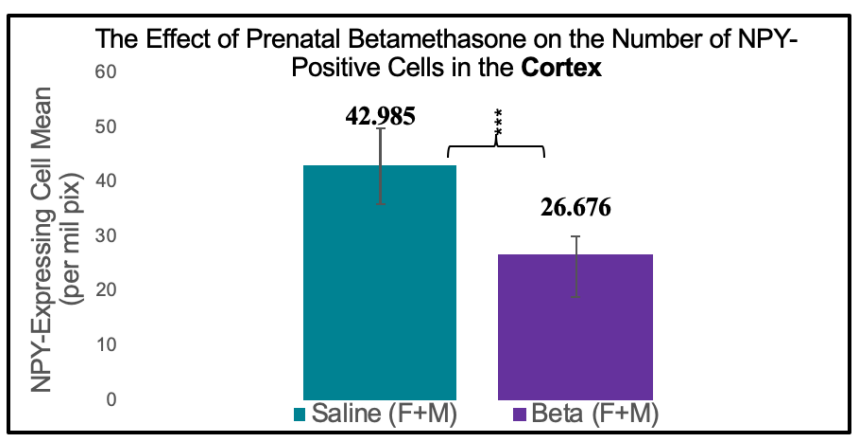

Figure 1: There is a significant decrease in the NPY Positive cells in the male and female group treated prenatally with betamethasone (shown in purple) compared to the control female brain treated prenatally with saline (shown in green) $(* * * 00001)$. This corresponds to the decrease in GABA neuron production in the offspring of stressed mothers treated with betamethasone.

The Effect of Prenatal Betamethasone on the Number of NPYPositive Cells in the Striatum

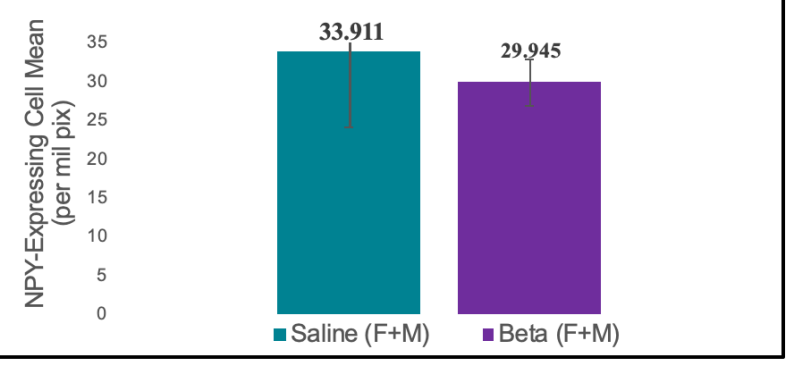

Figure 2: There is no significant decrease in the NPY-Positive cells in the male and female group treated prenatally with betamethasone (shown in purple) compared to the control female and male brain treated prenatally with saline (shown in green) $(\mathrm{p}=4295)$.

count in the saline/control model tissues, which did not receive the seizure-inducing treatment, was $42.985 \mathrm{NPY}$ cells/mil pix (Figure 1). There was a statistically significant difference be tween the two treatment groups seen in the NPY count of the cortex region of the brain, as evidenced by the two-way ANOVA ( $\mathrm{p}<.0001)$.

However, further statistical analysis revealed insignificant results in the striatum of the brain, where the mean count of NPY expressing cells in the experimental betamethasone group was $29.945 \mathrm{NPY}$ cells $\mathrm{mil} / \mathrm{pix}$ while the mean in the saline control was $33.911 \mathrm{NPY}$ cells mil/pix (Figure 2). The two-way ANOVA test revealed there was no statistically significant difference between the two treatment groups seen in the NPY count of the striatum region of the brain $(\mathrm{p}=.4295)$.

Overall, the resultant deficiency of NPY in only one region, the cortex, of the experimental rat brain confirms the significant decrease in the expression of GABA neurons in the offspring of stressed mother rats $(p<0.05)$ whereas the striatum did not show a significant decrease.

\section{GABAergic Count Based on Sex.}

Furthermore, an interaction two-way ANOVA analysis suggests a trend level difference between the presence of NPYexpressing cells between sexes in the cortex region of the brain, but not the striatum. Presence of NPY-expressing cells was significantly different among the two sexes in the cortex. Male brains had a greater amount of NPY markers in the cortex, with a mean count of $47.5 \mathrm{ctx} / \mathrm{mil}$ pix, than the cortex 
of the female group, with a mean cell count of $38.37 \mathrm{NPY}$ cells/mil pix (Figure 3). Females had a statistically significant decrease in NPY expression because they are more sensitive and more vulnerable to reacting with the stressor $(\mathrm{p}=0.0235)$. Clearly, there is a significant decrease in NPY expression in females, indicating that they have a greater decrease in GABA production than males, and are consequently more likely to develop epilepsy. It is important to note that the treatment was not the cause for this decrease in NPY expression in females,

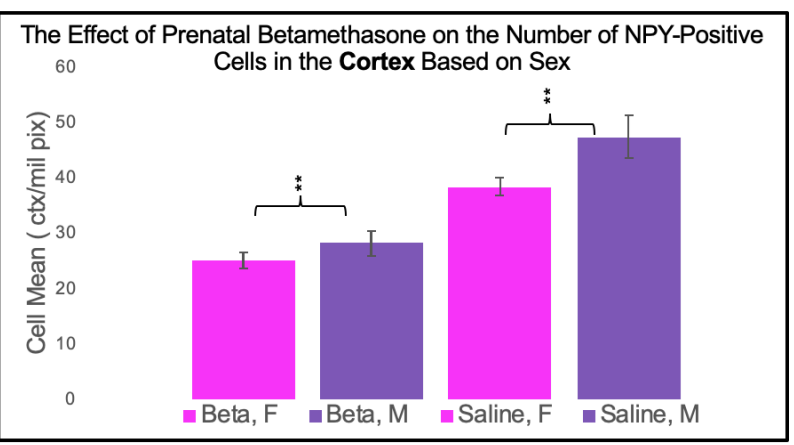

Figure 3: There is a significant decrease in the NPY-Positive cells in the cortex section of the female group (shown in pink) compared by the male group (shown in purple) in both treatment groups $(* * \mathrm{p}=.0253)$.

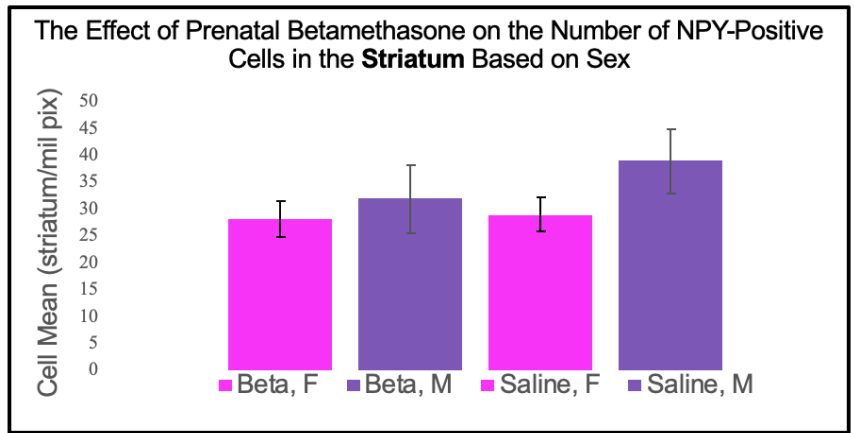

Figure 4: There is no significant difference in the NPY-Positive cells in the striatum section of either $\operatorname{sex}(\mathrm{p}=.1782)$.

considering that the significant decrease is seen even in the control group treated with saline. The females had a decrease in NPY expression in the cortex region of the brain, regardless of the treatment.

However, the same statistical result was not seen in the striatum region of the brain $(\mathrm{p}=.1782)$. The striatum region did not show a difference in NPY expression based on sex nor based on treatment (Figure 4).

This study demonstrates that prenatal exposure to betamethasone is associated with postnatal decreased expression of NPY in only the neocortex and not the dorsolateral striatum. In addition, we found that the cortex region has a decreased expression of NPY in females, regardless of the treatment group. In other words, only the cortex region showed that there was a significant difference in treatment group of the rats (with a decrease seen in the stressed offspring prenatally treated with betamethasone) and a significant difference based on sex (with an overall deficiency of NPY expression in females only). The striatum, however, showed no significant difference in treatment groups or either sex.
A decrease in NPY in the cortex may be detrimental to the activity of the rat brain considering a decrease in NPY also indicates a decrease in GABAergic neurons. As mentioned before, a deficiency in GABA immediately results in the increase in excitability in all mammals. One possible explanation for this decrease in expression in the offspring is that the betamethasone directly regulates the fetal NPY expression and the severe stress directly caused a decrease GABA expression.

\section{Transgenerational Epigenetic Inheritance.}

Another interesting possible explanation that connects to previous studies regarding the same topic focuses on the epigenome, where GABA production is regulated. The epigenome is the cellular material that surrounds the genome that contain epigenetic "marks" which signal to genes when to turn on or off. ${ }^{16}$ Environmental factors like diet, prenatal nutrition, and stress can all influence these epigenetic marks and make an imprint which passes to the next generation. This concept of transgenerational epigenetic inheritance can explain how stressed mothers can negatively influence the mental and physical development of the child. Thus, proving that the stress on the mother can change the epigenetic makeup of the brain, and these changes can have a negative effect on the direct offspring and future generations. Velisek et al 2006 conducted a study that used an animal model under stressful conditions to see how transgenerational inheritance can explain epilepsy being passed from mother to offspring. ${ }^{17}$ Similar to the results of this study, Velisek's results showed the betamethasone stressor altered epigenomic function via posttranslational modifications in the epigenome. This can mean adding or removing chemical tags that influence expression of GABA. ${ }^{18}$ The betamethasone decreased the number of GABAergic neurons in the brain, without obvious physical effects on the mother. However, the consequence of the stressor was experienced by the offspring of the mother rat. The stress was transferred onto her offspring and decreased the number of GABAergic neurons in the younger generation's brain. Some changes may be even transmitted to the second generation of offspring, which can be addressed in the future. This decrease of GABAergic neurons seen in both studies can be detrimental to the offspring because they can no longer finely regulate cell excitability, and may become more susceptible to having seizures and more likely to develop epilepsy. Essentially, the prenatal stressor betamethasone may have damaged the epigenome in both the mother and offspring.

The results of this study represent a significant advance over previous studies which focused on the role of prenatal exposure to betamethasone and NPY expression in anxiety. ${ }^{7}$ This study fills a gap in previous research by specifically looking at the effect of prenatal betamethasone on the production of GABAergic neurons in the neocortex and the striatum that would be consistent with susceptibility of the offspring to spasms. Statistical analysis between the two treatment groups (rats prenatally exposed to saline being the control, while rats prenatally exposed to betamethasone as the experimental) show the betamethasone caused a decrease in NPY, indicating a decrease in GABAergic neurons. 
This study concluded that the cortex only showed a significant difference in GABA production based on treatment group and sex, whereas the striatum region showed none. This result has been supported by previous studies that also link epilepsy with the cortex region of the brain. In fact, this can be explained by the fact that focal epileptic seizures are linked to abnormalities in three main brain regions: ipsilateral piriform cortex, temporal neocortex, and ventromedial prefrontal cortex. ${ }^{19}$ All three of these regions are part of the cortex region of the brain. This may be due to their enhanced sensitivity to prenatal betamethasone exposure. The sensitivity can be determined by differential genetic composition that regulate the concentration of glucocorticoid receptors. Glucocorticoids act via a number of direct and indirect routes that influence the developing epigenome. A change in concentration of these receptors can make offspring more vulnerable to changes caused by a stressor. ${ }^{20}$ Significant decrease in this neuronal population may contribute to enhanced propensity to development of spasms in the model of infantile spasms.

Interestingly, the study also found that sex plays a major role in the diagnosis of epilepsy. It found that females have a greater deficiency in GABA production, as evidenced by the significant lack of NPY-positive cells in females when compared to males. This can possibly be explained by the fact that females are more vulnerable to accepting the change the stressor induces. The alteration acquired from the mother is stronger in the daughter's cells and consequently, has the strength to decrease GABA expression in female cells. Thus, these results can support the conclusion reached in a previous study done by Christensen et. al $2005^{21}$ that found more women than men were diagnosed with idiopathic generalized epilepsy in two epilepsy populations.

These results can prove to be useful when applied to human treatment of health issues. Previous studies have shown that repeated antenatal administration of betamethasone is frequently used as a life-saving treatment in obstetrics. In fact, the trend in clinical practice has become to repeat the administration of synthetic corticosteroids, like betamethasone, in pregnant women at risk for premature delivery. However, no study has looked into the beneficial or detrimental effects of single antenatal corticosteroid administration. This study can serve as a starting point for future research considering how prenatal betamethasone can act as a stressor and lead to an increased likelihood of developing certain types of epilepsy. To put it simply, this research offers a possible explanation for the cause of epilepsy in patients. Other areas of future research can possibly look into how transgenerational inheritance of this stress can affect generations beyond the direct offspring, perhaps focusing on the grandchildren of mother rats. As mentioned before, it is definitely a possibility that sex can be a significant factor that can affect the GABAergic production in stressed rats.

\section{Future Research .}

Although the results of this study show significant progress from previous studies, there are ways to continue this study that can provide further evidence to confirm the link between epilepsy and genetics. In this experiment, the cortex and striatum were the only regions considered for GABA expression count, which may obscure the effects of betamethasone on other regions of the brain. Therefore, an experiment might be designed to examine the effect of betamethasone on GABA count in the hypothalamus region of the brain. Exploring hypothalamus region allows us to explain why it is a region that can cause many types of seizures in people with epilepsy and can progress the current study. In addition, the current study analyzes the brains of stressed rat mothers' direct offspring, but it should be investigated whether the decrease in GABA count is also seen in the grandchildren of the stressed mothers. This can help confirm that epilepsy is a hereditary disorder because of alterations made to the mother's epigenome due to stress.

\section{- Conclusion}

Prenatal exposure to betamethasone directly leads to decreased expression of NPY in the cortex of female and male brains. Since NPY serves as the marker for GABAergic neurons, a decrease in NPY indicates a decrease in the GABAergic neuron population in rats that were offspring of stressed mother rats. Ultimately, this indicates that the deficiency in GABAergic neurons may be the reason for impaired regulation of cell excitability, which increases the susceptibility to seizures. Prenatal betamethasone acted as a stressor and led to a decrease in GABA neurons in only the cortex region (not the striatum), so the hypothesis is refuted. This study expands on previous research, which primarily focused on repeated administration of betamethasone that actually proved to be beneficial to the mother rat. This study shows that single administration of betamethasone will induce great stress on the mother rat and will cause a decrease the production of GABA which is then passed onto the offspring. Chachua et al $2011^{4}$ established a rat model in her research that supports the conclusions of this study considering she was able to conclude that prenatal stress leads to an increase in spasms, which is explained by the decrease in GABA seen in this study. This research also proposes an explanation as to why the risk of epilepsy among people who have parents or siblings with the disorder is about $4 \%$ to $8 \%$, whereas the risk in the general population is $1 \%$ to $2 \%{ }^{22}$ The possible explanation is transgenerational epigenetic inheritance considering this study showed that stressed mothers give birth to epileptic offspring due to the modifications made in the epigenome that ultimately cause a decrease in GABA expression.

\section{- Methods}

\section{Overview.}

In order to see if one dosage of betamethasone would negatively affect the offspring, NPY was used as a marker to indicate either a decrease or increase in GABA neuron production. Since NPY is the marker for GABA, an increase of NPY signifies an increase in GABA neurons and a decrease in NPY signifies a decrease in GABA.

\section{Preparing the Brains .}

The brains used in this experiment were prepared by the mentor (Figure 5A). For the experimental group, the mentor injected the mother rat with prenatal betamethasone (2x 0.4 $\mathrm{mg} / \mathrm{kg}$ in $1 \mathrm{ml} / \mathrm{kg}$ of saline on gestational day 15; 08:00 and 
18:00), while the control group was injected with the saline. The experimental brains will be referred to as prebeta brains (short for prenatal betamethasone). The control brains will be referred to as presaline brains. The litter pups were sacrificed using euthanasia, and the brains were dissected and collected by the mentor. These brains were dissected from the rat bodies and preserved in a matrix solution in July 2019 and were kept in a $-80^{\circ} \mathrm{C}$ freezer for further use. Brains from both sexes of the two groups were gathered: one male prebeta, one male presaline, one female prebeta and one female presaline. These brains were perfused in a matrix solution and kept in a $-80^{\circ} \mathrm{C}$ freezer for future usage by the student when cutting.

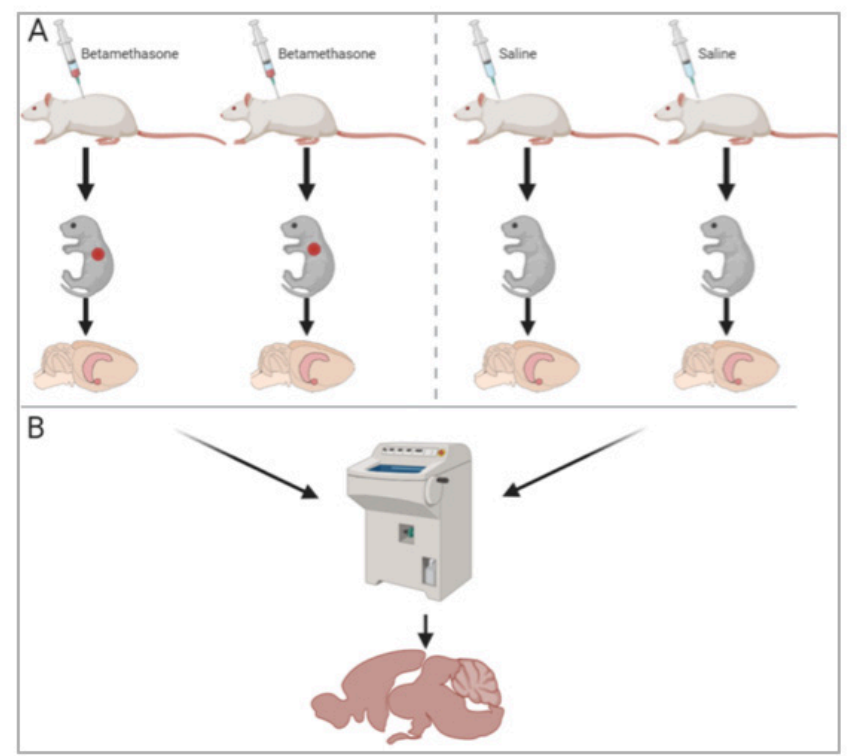

Figure 5: (A) Mentor created experimental and control group by injecting betamethasone and saline perspective and dissected brain out of newborn. (B) Student cut brains using cryostat to produce sagittal cuts of tissue.

\section{Cutting the Brains .}

First, the tissue from the four rat brains (two experimental, two control) had to be collected in order to stain for neuropeptide $\mathrm{Y}$. The tissue was acquired by using a cryostat, a machine capable of cutting very fine slices of frozen tissue (Figure 5B). Four brains were cut in total: male prebeta, male presaline, female prebeta and female presaline. The purpose was to have parallel data, making analysis easier and more consistent to compare. The brain was set up by gluing it down onto a steel holder using a matrix solution, which ensured the brain would be held in place. While waiting for that to freeze, the blade (which was in the freezer) was set up and positioned at an angle to get a smooth cut. The cryostat was set to cut slices of 40 micrometers, using the sagittal cut (cutting parallel with the plane of brain symmetry). Each slice of tissue was carefully picked up with a paintbrush and transferred into well plates filled with phosphate-buffered saline (PBS) to preserve and clean the tissue. The tissue collected in well plates was later used for immunohistochemistry. However, every fifth slice was transferred onto a gelatin microscope using the PBS solution to make it stick to the slide. These slides were used later for cresyl violet, a general staining technique for histological overview of the structures.

\section{CresylViolet.}

Some of the brain tissue can be folded and damaged. In order to have a reference and identify specific sections of the brain when scanning and analyzing the experimental tissue, the tissue that was collected onto gelatin microscope slides was used for cresyl violet. This is a process in which the tissue is generally stained purple to identify specific parts and structures of the brain when scanning and analyzing the experimental tissue. The slides with the fresh tissue were left overnight to dry. In order to clean slides, the tissues first had to be put into xylene for five minutes, followed by additional xylene for another five minutes. Then slides were transferred into $100 \%$ alcohol for three minutes, then $95 \%$ alcohol for three minutes and then $75 \%$ alcohol for three minutes. The purpose of this procedure is not only to clean and sterilize the tissue but dehydration, lipid removal and rehydration, which are all necessary for proper staining. The slides were then placed in cresyl violet for three minutes, allowing the tissue to be stained a purple color and the alcohol process was repeated to rinse the tissue.

\section{Immunohistochemistry.}

The process of immunohistochemistry (IHC) was used to stain the tissue for a specific antigen, using a corresponding antibody as a marker (Figure 6). ${ }^{23}$ The tissues that were collected in the well plates were washed with PBS three separate times, with ten minutes on the Rotoshaker (multiaction platform which gently shakes the tissues in the PBS) in between each wash. These washes ensured the tissues were cleaned and rehydrated with buffer solution. After washing and cleaning tissues, the blocking buffer was prepared using normal goat serum, BSA (bovine serum albumin), PBS and triton, which made the membranes in the tissue permeable for the antibody to enter. After letting it sit for 90 minutes, the blocking buffer made sure to prevent non-specific binding and ensured only the attachment on the NPY antigen. Once it was removed from the tissue, the primary antibody, antiNPY (the primary antibody at a 1:1000 concentration) was left incubating the tissue for 3 nights in order to tag the NPY antigen.

After the three nights, day 2 of the IHC process began by removing the primary antibody and washing the tissue three separate times in PBS for ten minutes each. The secondary antibody was diluted in biotinylated anti-rabbit serum, BSA and PBS. with a concentration of 1:200. As the tissue incubated in this solution for 60 minutes, it tagged onto the primary antibody and aided in marking the NPY antigen. Following the incubation with the secondary antibody, the avidinbiotin $(\mathrm{AB})$ solution was prepared with 1:1:2 concentration and PBS. After another 3 washes with PBS, the tissues were incubated in the $\mathrm{AB}$ solution for 60 minutes in order to bind to the biotin on the secondary antibody. Lastly, the tissues were washed three times again with PBS and were stained with diaminobenzidine (DAB), a general brown stain that binds to the avidin and made the primary antigen (NPY) molecules visible so that one can count each NPY-positive cell. 


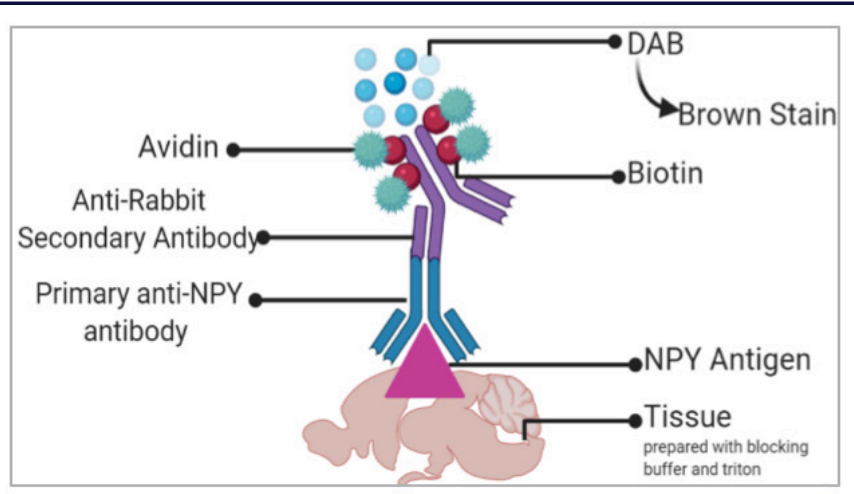

Figure 6: Process of immunohistochemistry includes layers of components interacting to stain a single antigen.

\section{Mounting and Cover Slipping .}

In order to view the stained tissues, they were transferred from the well plates onto $2 \%$ gelatin microscope slides using PBS and camel-hair paint brushes. Each group produced 4 slides of tissue (with roughly 5 slices per slide). In total, there were 82 slides. The tissues on these slides were preserved by using a cover slip made of glass that sealed off the tissue and ensured no bacteria/debris contaminated it. This process, referred to as cover slipping, involved using xylene (to clean the slides) and Permount (acting as a glue to stick coverslip onto the slide) in order to seal the slide and prevent any debris as well as air from entering.

\section{Scanning Slides .}

Before viewing the slides under the fluorescent light microscope, the slides must be cleaned using a blade to scrape excess dirt and washed with Permount and ethyl alcohol to clean. The slides were viewed at $10 \mathrm{x}$ magnification, specifically looking at the cortex and striatum regions of the brain to view the GABA neurons indicated by dark spots in the tissue. High quality snapshots were taken of the tissue using Olympus CellSens Dimension software on computer. Using a process called stitching, multiple magnified shots of the tissue were matched together to create a bigger $4 \times 1$ or $3 \times 1$ picture of the entire (cortex and striatum) region of the brain.

\section{Data Analysis .}

The images gathered from microscope scanning were analyzed using Fiji software. This program counted the GABA neurons in the cortex and striatum region of the brain. This process was carried out by creating a digital window for a specific section of each tissue with specific dimensions. Everything surrounding the window was then erased, leaving behind only the area inside the window to count NPY positive cells. The area measurement for the window was in pixels and the same measurement was used to make the same digital window for every tissue, which made sure that the data was equally distributed. These boxes highlighted the most concentrated of NPY positive cells which allows each individual dark spot to be counted. Each dark spot signified the presence of an NPY positive cell that was tagging onto a GABA neuron (Figure 7). All statistics in order to find the mean expression of NPY-positive cells across all four brains were transferred onto a Google Excel spreadsheet, which, included the brain group, section of the brain, the area of the section, and the count of neuron. Statistical analysis was done by the mentor who ran the twoway ANOVA tests that provided a $\mathrm{p}$-value. The $\mathrm{p}$-value was used to determine whether there was a statistically significant difference between the different brain tissues. A p-value less than or equal to .05 confirmed the data showed a statistically significant difference.

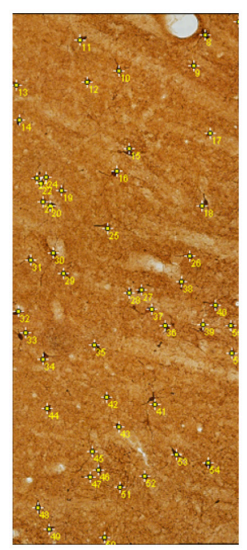

Figure 7: NPY positive cell count (56) of Male Striatum treated with betamethasone.

\section{Acknowledgement}

I am truly grateful for the aid from my mentor, Dr. Libor Velisek at New York Medical College who made the success of this project possible. I was lucky enough to have this opportunity granted by Dr. Velisek and his tremendous guidance made the completion of this project possible. In addition, I would like to thank my science research teacher, Jeff Wuebber, for his dedication and guidance with this report.

\section{References}

1. England M.J.; Liverman C.T.; Schultz A.M.; Strawbridge L.M. Epilepsy across the spectrum: Promoting health and understanding. Epilepsy \& Behavior 2012, 25, 266-276.

2. Epilepsy Foundation Home Page. https://www.epilepsy.com/learn/about-epilepsybasics/what-epilepsy (accessed August 14, 2019).

3. Stafstrom, C. E.; Carmant, L. Seizures and Epilepsy: An Overview for Neuroscientists. Cold Spring Harbor Perspect. Med. 2015, 5(6), a022426.

4. Chachua, T.; Yum, M.-S.; Velíšková, J.; Velíšek, L. Validation of the rat model of cryptogenic infantile spasms. Epilepsia 2011, 52(9), 1666-1677.

5. NYU Langone Medical Center / New York University School of Medicine. Largest study of epilepsy patients ever conducted reveals new and surprising genetic risk factors, 2013. ScienceDaily. www.sciencedaily.com/ releases/2013/08/130812103004.html

(accessed June 30, 2020).

6. Yum, M.S.; Chachua, T.; Velíšková, J.; Velíšek, L. Prenatal stress promotes development of spasms in infant rats. Epilepsia 2011, 53(3), e46-e49.

7. Price, M. G.; Yoo, J. W.; Burgess, D. L.; Deng, F.; Hrachovy, R. A.; Frost, J. D.; Jr Noebels, J. L. A triplet repeat expansion genetic mouse model of infantile spasms syndrome, $\operatorname{Arx}(\mathrm{GCG}) 10+7$, with interneuronopathy, spasms in infancy, persistent seizures, and adult cognitive and 
behavioral impairment. J. Neurosci. 2009, 29(27), 8752-8763.

8. Velísek, L.; Jehle, K.; Asche, S.; Velísková, J.; Model of infantile spasms induced by $\mathrm{N}$-methyl-D-aspartic acid in prenatally impaired brain. Ann. Neurol. 2007, 61(2), 109-119.

9. Khazipov, R.; GABAergic Synchronization in Epilepsy. Cold Spring Harbor Perspect. Med. 2016, 6(2), a022764.

10. Trevelyan, A.J.; Muldoon, S.F.; Merricks, E.M.; Racca, C.; Staley, K.J.; The role of inhibition in epileptic networks. J. Clin. Neurophysiol. 2015, 32(3), 227-234.

11. Miyamoto, H.; Tatsukawa, T.; Shimohata, A.; Yamagata, T.; Suzuki, T.; Amano, K.; Mazaki, E.; Raveau, M.; Ogiwara, I.; Oba-Asaka, A.; Hensch, T. K.; Itohara, S.; Sakimura, K.; Kobayashi, K.; Kobayashi, K.; Yamakawa, K. Impaired cortico-striatal excitatory transmission triggers epilepsy. Nature communications 2019, 10(1), 1917.

12. Velíšek, L.; Shang, E.; Velíšková, J.; Chachua, T.;

Macchiarulo, S.; Maglakelidze, G.; Wolgemuth, D. J.; Greenberg, D. A. GABAergic neuron deficit as an idiopathic generalized epilepsy mechanism: the role of BRD2 haploinsufficiency in juvenile myoclonic epilepsy. PloS one 2011, 6(8), e23656.

13. Velísková, J.; Velísek, L. Beta-estradiol increases dentate gyrus inhibition in female rats via augmentation of hilar neuropeptide Y. J. Neurosci. 2007, 27(22), 6054-6063.

14. The Revisionist. (2018, November 19). The Role of the Prefrontal Cortex in our Cognitive Function. The Revisionist. https://therevisionist.org/reviews/role-ofprefrontal-cortex-in-cognitive-function/ (accessed December, 2019).

15. Clynen, E.; Swijsen, A.; Raijmakers, M.; Hoogland, G.; \& Rigo, J. M. Neuropeptides as targets for the development of anticonvulsant drugs. Mol. Neurobiol. 2014, 50(2), 626-646. https://doi.org/10.1007/s12035-014-8669-x.

16. Cloud, J. Why Your DNA Isn't Your Destiny. Time Inc, 2010. http://content.time.com/time/magazine/ article/0,9171,1952313,00.html (accessed 10 Jul. 2020).

17. Velíšek, L. Prenatal Exposure to Betamethasone Decreases Anxiety in Developing Rats:

Hippocampal Neuropeptide Y as a Target Molecule. Neuropsychopharmacology 2006, 31(10), 2140-2149.

18. Kobow, K.; Reid, C.A.; Vliet, E.A.; Becker, A.J.; Carvill, G.L.; Goldman, A.M.; Hirose, S.; Lopes-Cendes, I.; Khiari, H.M.; Poduri, A.; Johnson, M.R.; Henshall, D.C.; Epigenetics explained: a topic "primer" for the epilepsy community by the ILAE Genetics/Epigenetics Task Force, 2020. Wiley Online

Website. https://onlinelibrary.wiley.com/doi/full/

19. Pedersen, M.; Curwood, E. K.; Vaughan, D. N.; Omidvarnia, A. H.; Jackson, G. D. Abnormal Brain Areas Common to the Focal Epilepsies: Multivariate Pattern Analysis of fMRI. Brain Connectivity 2016, 6(3), 208-215.

20. Moisiadis, V.; Matthews, S. Glucocorticoids and fetal programming part 2: mechanisms. Nat. Rev. Endocrinol. 2014, 10, 403-411.

21. Christensen, J.; Kjeldsen, M.J.; Andersen, H.; Friis, M.L.; Sidenius, P. Gender differences in epilepsy. Epilepsia 2005, 46(6), 956-960.

22. Institute of Medicine (US) Committee on the Public Health Dimensions of the Epilepsies, England,
M.J.; Liverman, C.T.; Schultz, A.M.; Strawbridge, L.M. Epilepsy Across the Spectrum: Promoting Health and Understanding. Washington (DC), National Academies Press (US) 2012. https://doi.org/10.17226/ 13379 (accessed on August 14, 2020)

23. Taylor, C. R.; Shi, S.; Barr, N. J. Techniques of Immunohistochemistry. Diagnostic Immunohistochemistry 2011,1-41..

\section{Author}

Akshara Koottala, the author of this paper and conductor of this research, is a rising senior at New Rochelle High School. She is interested in neuroscience and is passionate to establish a possible link between genetics and epilepsy. She plans to major in biology at the university she will attend. 